\title{
OS FLEBOTOMÍNEOS DA REGIÃO AMAZÔNICA, ESTRADA MANAUS - HUMAITA, ESTADO DO AMAZONAS, BRASIL (DÍPTERA: PSYCHODIDAE: PHLEBOTOMINAE).
}

\author{
Eloy G. CASTELLÓN', Jorge R. ARIAS ${ }^{2}$, Rui A. de FREITAS $^{1}$ \& Roberto D. \\ NAIFF'.
}

\begin{abstract}
RESUMO - Como parte do programa do laboratorio de Parasitología do INPA, na procura dos vetores e reservatorios da Leishmaniose, foi realizado durante o periodo de 1978 até 1981, um levantamento da fauna flebotomínica, em oito localidades da Rodovia BR-319 (Manaus-Humaita). Capturamos 57 espécies: 44 do gênero Lutzomyia e 13 de Psychodopygus. Os métodos de coleta usados foram: capturas nos troncos das árvores, isca humana e armadilhas luminosas, o método mais eficiente foi o da armadilha luminosa colocada a 6 metros de altura na floresta. As espécies mais comuns foram $\boldsymbol{L}$. antunesi, $\boldsymbol{P}$. chagasi e $\boldsymbol{P}$. ayrozai. Dados sobre a distribuição geográfica das espécies coletadas e a distribuição por coletas são também assinalados.
\end{abstract}

Palavras chaves: Flebotomineos, Psychodidae, distribuição, Amazônia.

Sand Flies of the Amazonian Region Manaus-Humaitá Highway, State of Amazonas, Brazil (Diptera: Psychodidade: Phlebotominae).

SUMMARY - As part of our ongoing program, searching for the vectors and reservoirs of Leishmania parasites, we conducted a survey of the sandfly fauna along the BR-319 (ManausPorto Velho) highway. Fifty seven species of sandflies were captured; 44 in the genus Lutzomyia and 13 Psychodopygus. Of the collecting methods utilized, CDC traps set $6 \mathrm{~m}$ above the forest floor proved to be the most effective, and the most frequently captured species were $\boldsymbol{L}$. antunesi, $\boldsymbol{P}$. chagasi and $\boldsymbol{P}$ ayrozai. We also present the geographical distribution of the different species collected.

Key-words: Sand flies, Psychodid, Distribution, Amazon.

\section{INTRODUÇÃO}

Embora a Leishmaniose Tegumentar Americana seja conhecida na Amazônia Brasileira, relativamente pouco se sabe sobre a fauna de flebotomíneos encontrados na margem direita do Río Solimões, e principalmente o possível ou possíveis vetores nessa área que se encontra em processo de colonização agroflorestal.

Devido à importância dos flebotomíneos na transmissão das Leishmanioses, este trabalho teve como objetivo o de conhecer as diferentes espécies ao longo da rodovia $\mathrm{Br}-319$ (área da estrada delimitada pelos Ríos Solimões e Madeira) no Estado do Amazonas, fazendo um levantamento em oito localidades, em 668 $\mathrm{Kms}$ de rodovia; fornecendo dados que permitam ampliar o conhecimento da distribuição geográfica, facilitando os estudos bioecológicos e epidemiológicos.

\section{MATERIAL E MÉTODOS}

Os locais de capturas, na mata amazônica corresponderam aos Kms 52,

1 Instituto Nacional de Pesquisas da Amazônia - INPA, Caixa Postal 478, CEP 69011-970, Manaus - AM.

2. Pan American Health Organization - OPAS, Setor de Embaixadas Norte, Lote 19, CEP 70.800, Brasília - DF. 
$100,150,235,275,400,500$ e $600 \mathrm{da}$ rodovia $\mathrm{BR}-319$, no trecho ManausHumaitá (Fig. 1) durante o periodo de três anos, de 1978 a 1981 , com coletas trimestrais. O estudo foi programado para realizar coletas equidistantes, a cada 50 $\mathrm{Km}$, no trecho de $668 \mathrm{Kms}$ de rodovia, com a finalidade de observar uma possível sucessão na distribuição horizontal, mais, devido ao difícil acesso e áreas extensamente alagadas, foram selecionadas apenas oito localidades.

As coletas foram realizadas com armadilhas luminosas tipo CDC; duas colocadas a $1 \mathrm{~m}$ e duas colocadas a $6 \mathrm{~m}$ de altura do solo da floresta, das 18:00 às 06:00 hs; captura nos troncos das árvores durante parte do dia e coletas com isca humana, em periodos noturnos. Em todas as localidades foram aplicadas as mesmas técnicas de coletas a fim de uniformizar a informação.

Embora não tenhamos esgotado todas as metodologías aplicáveis nas coletas deste tipo de inseto, usamos: as armadilhas CDC para observar a tendência da estratificação vertical das diferentes espécies, as coletas com isca humana para capturar as espécies com tendências antropófilas e na base de árvores para verificar as espécies de flebotomíneos num local de repouso durante o dia.

Os flebotomíneos foram preservados e montados de acordo com BIANCARDI et al. (1982) e YOUNG (1979).

$\mathrm{O}$ tratamento taxionômico segue a proposta de MARTINS, WILLIAMS \& FALCÃO (1978), com exceção de Psychodopygus, aquí considerado como gênero. A distribuição geográfica foi baseada nos trabalhos de MARTINS,
WILLIANS \& FALCÃO (1978), RYAN (1986) e YOUNG (1979).

\section{RESULTADOS}

\section{Captura em troncos de árvores}

A tabela 1 mostra as espécies de flebotomíneos capturados em troncos de árvores; 21 destas espécies pertencem ao gênero Lutzomyia e quatro espécies a Psychodopygus. As duas espécies mais abundantes nas coletas foram $L$. antunesi (Coutinho) e L. umbratilis (Ward \& Fraiha), embora duas outras espécies, $L$. dendrophila (Mangabeira) e L. spinosa (Floch \& Abonnenc) em menor número, tenham sido coletadas em todas as localidades.

\section{Isca Humana}

A tabela 2 mostra as espécies antropófilas; foram capturadas 13 espécies de Lutzomyia e 12 espécies de Psychodopygus; dessas, duas ainda não foram definitivamente identificadas: $L$. $s p$. e outra próxima a $P$. davisi, com o escutelo escuro. As espécies mais frequêntes foram $P$. ayrozai (Barreto \& Coutinho) e $P$. chagasi; $P$. paraensis (Costa Lima) embora em menor proporção, estava presente em todas as localidades, menos no $\mathrm{Km} \mathrm{235}$. As espécies do gênero Lutzomyia foram capturadas em muito menor proporção por esse método. As espécies do gênero Psychodopygus, coletadas em maior número, tem demostrado uma tendência antropófila maior.

\section{Armadilhas CDC}

Os resultados obtidos com o uso da armadilha luminosa $\mathrm{CDC}$, colocada 
a Im do solo na floresta, encontram-se na tabela 3 . Foram coletadas 28 espécies ¿ Lutzomyia e 9 espécies de Psychodopygus. As espécies coletadas em maior número foram $L$. antunesi e L. ubiquitalis (Mangabeira).

As espécies $L$. bacula (Martins, Falcão \& Silva), L. borrouli (Barreto \& Coutinho), L. flaviscutellata (Mangabeira), L. melloi (Causey \& Damasceno) e L. sherlocki (Martins, Silva \& Falcão) foram capturadas exclusivamente nas armadilhas CDC colocadas a $1 \mathrm{~m}$ de altura do solo.

A tabela 3, também apresenta os resultados obtidos em coletas com armadilhas luminosas, colocadas a $6 \mathrm{~m}$ de altura. Foram coletadas 36 espécies d Lutzomyia e 11 do gênero Psychodopygus. As espécies mais frequentemente coletadas e em maior número foram: $L$. antunesi $P$. chagasi. Outras espécies coletadas em menor proporção em todos os locais foram: $L$. yuilli yuilli (Young \& Porter), $P$. ayrozai e $P$. davisi (Root). Quinze espécies: L. chassignetti (Floch \& Abonnenc), L. choti (Floch \& Abonnenc), L. dreisbachi (Causey \& Damasceno), L. flabellata (Martins \& Silva), L. gomezi (Nitzulescu), L. olmeca olmeca (Vargas \& Díaz-Nájera), e $L$. richardwardi (Ready \& Fraiha), $L$. rondoniensis (Martins, Falcão \& Silva), L. rorotaensis (Floch \& Abonnenc), L. runoides (Fairchild \& Hertig), $L$. shannoni (Dyar), L. umbratilis, e $L$. wagleyi (Causey \& Damasceno), $P$. hirsutus hirsutus (Mangabeira) e $P$. wellcomei (Fraiha, Shaw \& Lainson) foram coletadas exclusivamente nas armadilhas colocadas a $6 \mathrm{~m}$ de altura.

Distribuição geográfica por: subgêneros, grupos e series.

\section{Gênero Lutzomyia Grupo Cruciata}

Está representada por duas espécies: L. gomezi $\left(30^{*}\right)$, espécie de ampla distribuição desde o México até o Paraguai, coletada nos Kms. 100 e 275; no Brasil é achada em toda a Amazônia legal; L.sherlocki $\left(10^{7}\right)$ achada no $\mathrm{Km} 600$. Esta espécie foi encontrada na Amazônia Peruana e no Brasil, nos Estados do Acre, Rondônia e Mato Grosso.

\section{Grupo Gasparviannai}

Com duas espécies aqui citadas, L. flabellata $\left(10^{7}\right)$ no $\mathrm{Km} 100$; espécie com distribuição muito restrita, correspondente aos Estados de Acre e Rondônia; L spathotrichia (Martins, Godoy \& Silva) $\left(40^{*}\right)$ no $\mathrm{Km} 100$, tambén com estreita distribuição, encontrada somente no Brasil, no Estado de Roraima e no Estado do Amazonas.

\section{Grupo walkeri}

Aqui representada por $L$. walkeri (Newstead) (110 $15 \%$, espécie coletada nos Kms 52, 100, 275 e 600. Com distribuição desde o Panamá até o Paraguai e Trinidad. No Brasil tem sido coletada no Acre, Goiás, Mato Grosso, Pernambuco e Rondônia.

\section{Grupo saulensis}

Capturadas duas espécies, $L$. saulensis (Floch \& Abonnenc) $\left(40^{\pi}\right)$, coletada em todas as áreas exceto nos $\mathrm{Kms}$ 
150 e 500.

Distribuida desde Centro América até o Perú, passando pela Guiana. No Brasil tem sido achada na Amazônia legal e Goiás. L. wilsoni (Damasceno \& Causey) (11 $\left.\sigma^{7}\right)$ coletada nos Ks 100 e 275. Espécie de estreita distribuição, somente assinalada no Brasil nos Estados do Amazonas e Rondônia. Foram capturadas ainda 115 que poderiam ser de qualquer dessas espécies, já que de acordo com Martins et al. (1983) são indistinguíveis.

\section{Subgênero Pintomyia}

Representado por L. spinosa (Floch \& Abonnenc) (97 $\sigma^{\pi}$ e 34\%), coletadas em todas as localidade onde se desenvolveu este trabalho; espécie encontrada no Panamá, Colômbia, Guiana Francesa e no Brasil distribuida em toda a Amazônia.

\section{Subgênero Pressatia}

Com uma espécie aqui citada: $L$. choti $\left(3 \sigma^{\circ}, 1\right.$ \% $)$ dos Kms 100, 275, distribuida no Brasil pelos Estados do Acre, Pará, Maranhão, Espírito Santo, Pernambuco e Bahia. Fora do Brasil, achada na Guiana Francesa e Perú.

\section{Subgênero Evandromyia}

Série infraspinosa

Com duas espécies aqui citadas: $L$. bourroli $\left(40^{x}, 3\right.$ ㅇ), coletada nos Kms 52 e 275; espécie amplamente distribuida no Brasil, nos Estados do Acre, Amazonas, Goiás, Mato Grosso, Maranhão, Pará e São Paulo; e $L$. infraspinosa (Mangabeira) (40", 10ㅇ), capturadas nos Kms 52, 100 e 400 referida na Guiana Francesa e Suriname. No Brasil, é citada para o territorio do Amapá e Estado do Pará e Rondônia.

\section{Série Monstruosa}

L. monstruosa (Floch \& Abonnenc) $\left(40^{\star}, 5\right.$ \% ), nos Kms 52, 100 e 235; achada nos Estados do Acre, Amazonas, Maranhão, Pará e Rondônia, além do Estado do Amapá. Fora do Brasil foi encontrada na Guiana Francesa.

\section{Subgênero Viannamyia}

Aqui representado por três espécies. L. fariasi (Damasceno, Causey \& Arouck) $\left(30^{7}\right)$, coletada somente no $\mathrm{Km} 52$, espécie citada da Guiana Francesa e no Brasil, nos Estados do Amazonas e Pará. $L$. furcata (Mangabeira) (190", 28ㅇ), Kms 52, 100, 235, 275 e 400 ; com distribuição em Honduras, Panamá, Colômbia, Guiana Francesa e Perú. No Brasil, é achada em toda a Amazônia legal. L. tuberculata (Mangabeira) (37ㅇ) nos Kms 52, 100, 275, 400 e 600; espécie conhecida na Amazônia Legal e. fora do Brasil, no Panamá, Colombia, Guiana Francesa e Suriname.

\section{Subgênero Psathyromyia}

Representado aqui por 5 espécies: $L$. abonnenci (Floch \& Chassignet) $\left(50^{7}\right)$ dos Kms 52, 100 e 275; citada do Panamá, Colômbia, Guiana Francesa e Perú. No Brasil: Na Amazônia Legal e no Ceará. L. dendrophila (206), distribuida na Colômbia e Bolivia. No Brasil, na Amazônia Legal. Espécie coletada em todos os locais de trabalho. L. lutziana (Costa Lima) (6o 
e 5ㅇ); amplamente distribuida no Brasil na Amazônia Legal, Minas Gerais, Rio de Janeiro e São Paulo; capturada nos Kms 100, 275 e 600. L. scaffi (Damasceno \& Arouck) ( $\left.310^{\circ}\right)$, coletadas em quase todas as áreas, menos nos Kms 150 e 235; espécie citada do Perú e na Amazônia Brasileira. L. shannoni $\left(14 \sigma^{\top}\right)$ foi achado em todos os locais menos nos Kms 500 e 600; espécie com ampla distribuição desde a América do Norte até a Argentina, igualmente em todo o Brasil.

\section{Subgênero Trichopygomyia}

Quatro espécies estão representadas neste trabalho: $L$. dasipodogeton (Castro) $\left(30^{*}, 1 \%\right.$ ), dos Kms 100 e 275. Espécie encontrada anteriormente no Brasil nos Estados do Pará e Rondônia e no Amapá. L. longispina (Mangabeira) (51 $0^{\star}$, 23ㅇ), espécie capturada nos Kms 52, 100 , 150,275 e 500. Distribuida na Colômbia e Venezuela; no Brasil no Amazonas, Bahia, Minas Gerais, Pará, Pernambuco e Rondônia. L. rondoniensis $\left(30^{7}\right)$, capturada no $\mathrm{Km} \mathrm{275}$, anteriormente coletada unicamente na sua localidade tipo. $L$. wagleyi $\left(40^{\top}\right)$ nos $\mathrm{Kms} 275$ e 400 com distribuição muito restrita. Conhecida somente no Estado do Amazonas.

\section{Grupo brasiliensis}

L. aragaoi (Costa Lima) ( $280^{\circ}$, 20) achada nos Kms 52, 100, 275, 500 e 600, conhecida desde o Panamá até o Paraguai. No Brasil em toda a Amazônia, além dos estados de Minas Gerais, Paraná e Pernambuco. $L$. runoides $\left(40^{7}\right)$ coletada nos Kms 235 ,
275 e 400. Distribuida desde a América Central até o Brasil. Na Amazônia nos Estados do Acre, Amazonas e Rondônia, achada também em Minas Gerais.

\section{Grupo chassigneti}

L. chassigneti $\left(10^{7}\right)$, exemplar do $\mathrm{Km}$ 100. Conhecida no Brasil em Rondônia. Fora do Brasil, referida na Guiana Francesa.

\section{Subgênero Trichophoromyia}

Representada aqui por cinco espécies: L. auraensis (Mangabeira) $\left(950^{\circ}\right)$,espécie coletada em todas as áreas

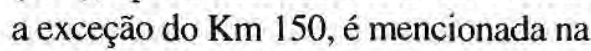
Colômbia, Perú e Bolivia. No Brasil, no Acre, Amazonas, Pará e Rondônia. $L$. brachipyga (Mangabeira) $\left(50^{*}, 4 \%\right)$, achada nos Kms 52, 100 e 235. Citada na Guiana Francesa. No Brasil, no Amazonas, Pará e Rondônia. L. melloi $\left(90^{x}\right)$, coletada somente no $\mathrm{Km} 275$. Espécie achada unicamente no Brasil, no Amazonas. L. octavioi (Vargas) (390'), achada nos Kms 52, 100, 235, 275 e 500. Citada no Penu e no Brasil: no Amazonas e Rondônia. L. ubiquitalis (3730*, 115 \%) Venezuela e Guiana Francesa; no Brasil, na Amazônia Legal.

\section{Subgênero Nyssomyia}

Com sete espécies neste trabalho: L. anduzei (Rozeboom) (430, 125ㅇ), coletada nos Kms S2, 100, 235, 275, 400 e 600. Amplamente distribuida desde a Costa Rica até o Perúe na Amazônia Brasileira. L. antunesi $\left(6800^{\star}, 1794\right.$ 우), espécie predominante em todos os locais 
de coletas. Distribui-se desde a Colômbia, Guiana Francesa, Perú, Trinidad, Venezuela; na Amazônia Brasileira foi achada nos Estados do Amazonas, Pará, Rondônia, Roraima, Acre, Mato Grosso, Minas Gerais, Paraná e Pernambuco. L. flaviscutellata $\left(7 \sigma^{x}, 48\right.$ \%), capturada em todos os locais, menos no $\mathrm{Km} \mathrm{150.} \mathrm{Conhecida} \mathrm{na}$ Colômbia, Perú, Guiana Francesa, Suriname, Trinidad, Tobago e Venezuela e no Brasil; achada na Amazônia Brasileira, Ceará, Espírito Santo, Minas Gerais e Rio de Janeiro. L. olmeca (sensu lato) (140, 17우) foi coletada em todas as áreas exceto no $\mathrm{Km} 150$. Distribuida desde o México, Centro américa, Colombia, Venezuela e no Brasil, nos arredores de Manaus, Rio Trombetas e no Acre. L. richardwardi $\left(130^{7}\right)$, aqui só não coletada dos $\mathrm{Kms}$ 150 e 235 . Conhecida somente do Brasil dos Estados do Acre, Pará e Rondônia. L. umbratilis $\left(143 \sigma^{*}, 248\right.$ \% $)$ e $L$ yuilli yuilli $\left(50^{x}, 194\right.$ 우) frequente em todos os locais de coleta desta área. Distribuida desde a Colômbia até o Perú e no Brasil em toda a Amazônia.

\section{Grupo dreisbachi}

L. dreisbachi $\left(20^{\pi}\right)$ espécie coletada no $\mathrm{Km} 500$. Assinalada na Guiana Francesa, Colômbia e no Brasil: Amazonas e Rondônia.

\section{Subgênero Helcocyrtomyia}

\section{Grupo oswaldoi}

L. rorotaensis $\left(20 \sigma^{\circ}\right)$ capturada nos Kms 100, 275 e 600. Distribuida desde o Panamá até a Guiana Francesa e no Brasil em quase toda a Amazônia. L. trinidadensis (Newstead), com um só exemplar fêmea coletada no $\mathrm{Km}$ 400. Com ampla distribuição do México até Bolivia, e no Brasil em toda a Amazônia, Ceará Goiás, Minas Gerais e Pernambuco.

\section{Lutzomyia nao agrupadas}

L. bacula $\left(20^{\pi}\right)$ coletadas nos Kms 100 e 275; conhecida somente no Brasil nos Estados de Goiás e Rondônia. L. nordestina (Mangabeira) $\left(160^{*}, 26\right.$ \% ) só não coletada no $\mathrm{Km}$ 235; com ampla distribuição desde o Panamá até o Perú e Trinidad; no Brasil, em toda a Amazônia, Ceará, Goiás, Minas Gerais e Pernambuco.

\section{Gênero Psychodopygus}

\section{Série chagasi}

Representada por quatro espécies $P$. chagasi $\left(2210^{\circ}, 1426 \%\right)$ a segunda espécie com maior número de individuos no total de capturas e frequente em todas as áreas. Amplamente distribuida na Colômbia e Perú; e no Brasil, em quase toda a Amazônia legal. P. corossoniensis (Le Pont \& Pajot) (10 113 \& espécie coletada por nós em todas ás áreas exceto nos Kms 150 e 400. Com estreita distribuição; citada na Guiana Francesa, e no Brasil, no Amazonas e Rondônia. P. guyanensis (40", 134 \%) coletada frequentemente em todas as áreas de trabalho, assinalada em Belize, e da Colômbia ao Perú; no Brasil, além da Amazônia foi achada também em São Paulo. P. wellcomei $\left(40^{7}\right)$ foram coletados exemplares nos Kms 52, 100 e 235. Assinalada no Pará e Ceará, as fêmeas são muito semelhantes as fêmeas de $P$. chagasi. 


\section{Série panamensis}

Está aqui representado por oito espécies. $P$. amazonensis (Root) $\left(20^{*}, 8 \%\right)$ coletada nos Kms 52, 100 e 500; espécie citada em Trinidad e Perú; no Brasil, em toda a Amazônia legal. P. ayrozai (1850", 951 +). A terceira espécie com maior incidência encontrada emtodos os locais de coleta, distribuida desde o Panamá ao Brasil; frequente em toda a Amazônia assim como em Espírito Santo, Minas Gerais, Rio de Janeiro e São Paulo. $L$. carrerai carrerai $\left(240^{\star}, 112\right.$ \%) espécie comum em todas as áreas trabalhadas. Citada na Colômbia, Perú e Ecuador. No Brasil, achada em toda a Amazônia, além da Bahia e Rio de Janeiro. P. claustrei (Abonnenc, Leger \& Fauran) (58 $0^{\star}, 58$ 우) espécie não coletada no $\mathrm{Km} 150$. Achada na Guiana Francesa e no Brasil, no Amazonas e Rondônia. P. davisi (2280",
668우) achada em todos os lócais de coleta, com distribuição bastante ampla na Colômbia e Bolivia e no Brasil em toda a Amazônia, Goiás, Espírito Santo, Minas Gerais e Rio de Janeiro. P. davisi (escuro) (11 \&) espécie em estudo, achada nos Kms 52,275 e 400 . P. hirsutus hirsutus (9우) coletada em todos os locais, menos nos $\mathrm{Kms} 150$ e 500. Amplamente distribuida no Brasil, em toda a Amazônia legal. $P$. paraensis $\left(490^{\star}, 322\right.$ ㅇ) espécie comum em todas as áreas; citadas da Colômbia, Perú e Suriname. No Brasil, coletadas no Amapá, Amazonas, Pará e Rondônia.

\section{Série arthuri}

Representada aqui por uma espécie, P. bispinosus (Fairchild \& Hertig) (4우) espécie coletada nos Kms 52, 235 e 500. Citada da América Central, Colômbia e Ecuador. No Brasil, no Amazonas, Amapa, Mato Grosso e Pará.

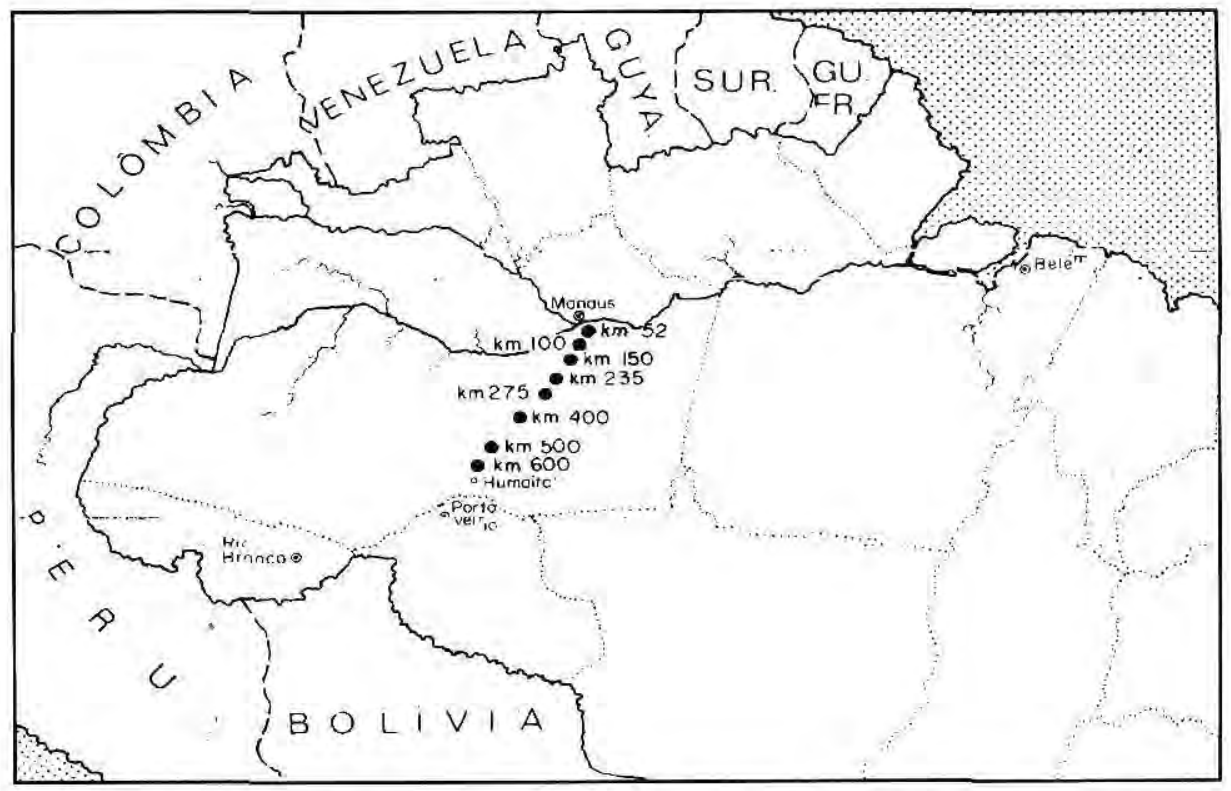

Figura 1. Mapa do Estado do Amazonas, mostrando as localidades (kms) onde foram coletados os flebótomos. 
Tabela 1. Flebotomineos coletados nos troncos das árvores, em oito localidades da Rodovia BR319 (Manaus-Humaitá/AM).

\begin{tabular}{|c|c|c|c|c|c|c|c|c|}
\hline ESPÉCIES & $\mathrm{Km} 52$ & $\mathrm{Km} 100$ & $\mathrm{Km} 150$ & $\mathrm{Km} 235$ & Km 275 & $\mathrm{Km} 400$ & $\mathrm{Km} 500$ & $\mathrm{~km} 600$ \\
\hline Lutzomyia abonnenci & 2 & 1 & & & & & & \\
\hline L. anduzei & & 3 & & & 3 & & & \\
\hline L. antunesi & 124 & 155 & 13 & 5 & 7 & 40 & 1 & \\
\hline L. aragaoi & 1 & & & & & & & \\
\hline L. dendrophila & 7 & 60 & 6 & 6 & 62 & 34 & 5 & 16 \\
\hline L. fariasi & 3 & & & & & & & \\
\hline L. flaviscutellata & 1 & 2 & & & & 10 & & \\
\hline L. furcata & 4 & 3 & & & 2 & 1 & & \\
\hline L. gonezi & & 1 & & & & & & \\
\hline L. longispina & 4 & 16 & & & & & & \\
\hline L. rorotaensis & & 17 & & & & & & \\
\hline L. saulensis & 2 & & & & & & & \\
\hline L. scaffi & 2 & 5 & & & 9 & 10 & 3 & 2 \\
\hline L. shannoni & 12 & 19 & 3 & 3 & 71 & 31 & & \\
\hline L. spathotrichia & & 4 & & & & & & \\
\hline L. spinosa & 5 & 15 & 10 & 9 & 60 & 4 & 5 & 5 \\
\hline L. tuberculata & 1 & & & & 2 & & & \\
\hline L. ubiquitalis & & & 1 & 1 & 5 & 1 & & 12 \\
\hline L. umbratilis & 1 & 155 & 4 & 2 & 38 & 6 & & \\
\hline L. walkeri & 1 & & & & & & & \\
\hline L. yuilli yuilli & & & 1 & & 1 & & & \\
\hline P. ayrozai & & & & & 3 & 2 & & \\
\hline P. chagasi & & & & 3 & 1 & 19 & 4 & 2 \\
\hline P. davisi & 2 & 2 & & & & 2 & 2 & 1 \\
\hline P. paraensis & 1 & & & & & 1 & & \\
\hline
\end{tabular}


Tabela 2. Flebótomos coletados atacando o homem em oito localidades da BR-319 (Manaus-Humaitá/AM).

\begin{tabular}{|c|c|c|c|c|c|c|c|c|}
\hline ESPÉCIES & $\mathrm{Km} 52$ & $\mathrm{Km} 100$ & $\mathrm{Km} 150$ & $\mathrm{Km} 235$ & $\mathrm{Km} 275$ & $\mathrm{Km} 400$ & $\mathrm{Km} 500$ & $\mathrm{~km} 600$ \\
\hline L. anduzei & & 2 & & & & & & 1 \\
\hline L. antunesi & 5 & 8 & & & 1 & 3 & & 1 \\
\hline L. aragaoi & & 1 & & & & & & \\
\hline L. flaviscutellata & 4 & & & 4 & 6 & 5 & 1 & 1 \\
\hline L. infraspinosa & & & & & & 1 & & \\
\hline L. nordestina & 1 & 4 & & & & & & \\
\hline L. richardwardi & 1 & 1 & & & & & 1 & 2 \\
\hline L. spinosa & 2 & & & & 2 & & & \\
\hline L. trinidadensis & & & & & & 1 & & \\
\hline L. ubiquitalis & & 1 & & & 1 & & & \\
\hline L. umbratilis & & 22 & & & 57 & 5 & 1 & 2 \\
\hline L. yuilli yuilli & & & 10 & & 74 & 6 & 6 & \\
\hline L.sp. & 2 & 1 & & 1 & 5 & & & \\
\hline P. amazonensis & & 3 & & & & & & \\
\hline P. ayrozai & 33 & 94 & 110 & 16 & 140 & 88 & 134 & 2 \\
\hline P. bispinosus & 1 & & & 2 & & & 1 & \\
\hline P. carrerai carrerai & 37 & 12 & & & 1 & 2 & 5 & \\
\hline P. chagasi & 2 & 11 & 45 & 26 & 206 & 89 & 121 & 58 \\
\hline P. claustrei & 6 & 3 & & & 5 & 5 & 9 & 3 \\
\hline P. corossoniensis & & 2 & & 63 & 2 & & 5 & 19 \\
\hline P. davisi & 22 & $œ 2$ & 1 & 5 & 9 & 41 & 78 & 22 \\
\hline P. davisi (escuro) & 3 & & & & 1 & 7 & & \\
\hline P. guyanensis & & 11 & 35 & 17 & 4 & 3 & 7 & \\
\hline P. hirsutus hirsutus & & 1 & & & 1 & 1 & & 1 \\
\hline P. paraensis & 5 & 40 & 34 & & 42 & 30 & 34 & 7 \\
\hline
\end{tabular}


Tabela 3. Espécies de flebotomineos capturados com armadilhas luminosas tipo CDC, colocadas a $1 \mathrm{~m}$ e $6 \mathrm{~m}$ de altura do solo na floresta em oito localidades da Rodovia BR-319 (Manaus-Humaitál AM).

\begin{tabular}{|c|c|c|c|c|c|c|c|c|c|c|c|c|c|c|c|c|}
\hline \multirow{3}{*}{ ESPÉCIES } & \multirow{2}{*}{\multicolumn{2}{|c|}{$\begin{array}{l}\mathrm{Km} 52 \\
\mathrm{CDC}\end{array}$}} & \multirow{2}{*}{\multicolumn{2}{|c|}{$\begin{array}{r}\mathrm{Km} 100 \\
\text { CDC }\end{array}$}} & \multirow{2}{*}{\multicolumn{2}{|c|}{$\begin{array}{r}\mathrm{Km} 150 \\
\mathrm{CDC}\end{array}$}} & \multirow{2}{*}{\multicolumn{2}{|c|}{$\begin{array}{r}\mathrm{Km} 235 \\
\mathrm{CDC}\end{array}$}} & \multirow{2}{*}{\multicolumn{2}{|c|}{$\begin{array}{r}\mathrm{Km} 275 \\
\mathrm{CDC}\end{array}$}} & \multirow{2}{*}{\multicolumn{2}{|c|}{$\begin{array}{r}\mathrm{Km} 400 \\
\text { CDC }\end{array}$}} & \multirow{2}{*}{\multicolumn{2}{|c|}{$\begin{array}{r}\mathrm{Km} 500 \\
\mathrm{CDC}\end{array}$}} & \multirow{2}{*}{\multicolumn{2}{|c|}{$\begin{array}{r}\mathrm{Km} 600 \\
\mathrm{CDC}\end{array}$}} \\
\hline & & & & & & & & & & & & & & & & \\
\hline & 1 & 6 & 1 & 6 & 1 & 6 & 1 & 6 & 1 & 6 & 1 & 6 & 1 & 6 & 1 & 6 \\
\hline Lutzomyia abonnenci & & & & & & & & & 1 & 1 & & & & & & \\
\hline L. anduzei & & 8 & 53 & 88 & & & & 1 & 1 & 6 & & 2 & & & & \\
\hline L. antunesi & & 68 & 301 & 945 & 3 & 4 & 1 & 6 & 5 & 42 & 2 & 14 & 1 & 6 & & \\
\hline L. aragaoi & & 3 & 3 & 3 & & & & & & 33 & & & 1 & & 2 & 1 \\
\hline L. auraensis & 1 & 5 & 6 & 8 & & & & 2 & 3 & 35 & 2 & 17 & 1 & 14 & 1 & \\
\hline L. bacula & & & 1 & & & & & & 1 & & & & & & & \\
\hline L. borrouli & 1 & & & & & & & & 6 & & & & & & & \\
\hline L. brachipyga & & 2 & 1 & 5 & & & 1 & & & & & & & & & \\
\hline L. chassigneti & & & & 1 & & & & & & & & & & & & \\
\hline L. choti & & & & 1 & & & & & & & & & & & & \\
\hline L. dasipodogeton & & & & 2 & & & 2 & & & & & & & & & \\
\hline L. dendrophila & 1 & & & 2 & & & & & 1 & 1 & 1 & & & & 2 & 2 \\
\hline L. dreisbachi & & & & & & & & & & & & & & 2 & & \\
\hline L. flabellata & & & & 1 & & & & & & & & & & & & \\
\hline L. flaviscutellata & 3 & 6 & & & & & 1 & & & & 1 & & 3 & & 7 & \\
\hline L. furcata & & 4 & 10 & 22 & & & 1 & & & & & & & & & \\
\hline L. gonezi & & & & & & & & & & 2 & & & & & & \\
\hline L. infraspinasa & 1 & & 11 & 1 & & & & & & & & & & & & \\
\hline L. longispina & & 10 & 14 & 27 & & 1 & & & & 1 & & & 1 & & & \\
\hline L. lutziana & & & 1 & 3 & & & & & & 1 & & & & & & 6 \\
\hline L. melloi & & & & & & & & & 9 & & & & & & & \\
\hline L. monstruosa & 1 & 2 & 3 & 1 & & & 2 & & & & & & & & & \\
\hline L. nordestina & & 2 & 2 & 5 & & 1 & & & & 4 & & & 6 & & 3 & \\
\hline L. octavioi & & & 2 & & & & 9 & & 4 & & & 6 & 3 & 7 & & 1 \\
\hline L. olmeca (s. latu) & & 5 & 3 & & & & & 8 & & 2 & & 6 & & 3 & & 4 \\
\hline L. nichardwardi & & & 1 & & & & & & & 3 & & 3 & & & & 1 \\
\hline L. rondoniensis & & & & & & & & & & 3 & & & & & & \\
\hline L. rorotaensis & & & 1 & & & & & & & 1 & & & & & & 1 \\
\hline L. runoides & & & & & & & & 1 & & 2 & & 1 & & & & \\
\hline L. saulensis & 4 & 8 & 17 & 68 & & & & 2 & 1 & 9 & & 4 & & & & 4 \\
\hline L. shannoni & & & & & & & & 1 & & 1 & & & & & & \\
\hline L. sherlocki & & & & & & & & & & & & & & & 1 & \\
\hline L. $s p$. & & 6 & 100 & 16 & & 1 & & & & & & & & 2 & & \\
\hline L. spinosa & 5 & 2 & 2 & & & & 1 & 2 & & & & 1 & & & 1 & \\
\hline L. tuberculata & 1 & 1 & 21 & 3 & & & & 3 & 1 & & & 1 & & & 1 & \\
\hline L. ubiquitalis & & 16 & 44 & 27 & 2 & & 4 & 48 & 31 & 138 & 5 & 18 & 4 & 46 & 20 & 73 \\
\hline L. umbratilis & & & & 53 & & 1 & & . & & 26 & & 3 & & 3 & & 7 \\
\hline L. wagleyi & & & & & & & & & & 2 & & 2 & & & & \\
\hline L. walkeri & 6 & 11 & & 6 & & & & & & 1 & & & & & & 1 \\
\hline
\end{tabular}


continuação Tabela 3.

\begin{tabular}{|c|c|c|c|c|c|c|c|c|c|c|c|c|c|c|c|}
\hline L. wilsoni & & 2 & & & & & 1 & & & & & & & & \\
\hline L. yuilli yuilli & & 7 & & 18 & 4 & 12 & 4 & 12 & & 1 & & & & & \\
\hline P. amazonensis & 2 & 2 & & 1 & & & & & & & & & 2 & & \\
\hline P. ayrozai & 7 & 21 & 14 & 36 & 1 & 12 & 6 & 54 & 320 & 7 & 26 & & 6 & 1 & 3 \\
\hline P. carrerai carreral & 3 & 28 & 1 & 2 & 1 & & & 8 & 2 & & 1 & 1 & 11 & & 21 \\
\hline P. chagasi & & 84 & 1 & 74 & & 10 & 5 & 60 & 485 & 8 & 114 & 2 & 187 & 1 & 29 \\
\hline P. claustrei & & 2 & 3 & 9 & & & 1 & & 15 & & 11 & & 23 & & 21 \\
\hline P. corossoniensis & & 9 & & 1 & & & & 4 & 9 & & & & & & \\
\hline P. davisi & 13 & 123 & 51 & 174 & & 2 & 8 & 32 & 124 & 2 & 54 & 2 & 39 & 7 & 36 \\
\hline P. guyanensis & & 1 & 3 & 13 & & & & & 6 & & & 1 & 25 & & 12 \\
\hline P. hirsutus hirsutus & & 3 & & 1 & & & 1 & & & & & & & & \\
\hline P. paraensis & 56 & & 1 & 12 & & & 4 & 1 & 101 & & & 2 & & & \\
\hline P. wellcomei & 1 & & & 2 & & & 1 & & & & & & & & \\
\hline
\end{tabular}

\section{DISCUSSÃO}

Em geral, as três espécies mais comuns foram $L$ antunesi, $\quad P$. chagasi $\mathrm{e}$ $P$. ayrozai e as menos coletadas, $L$. chassigneti, $L$ flabellata, $L$. sherlocki $\mathrm{e}$ L. trinidadensis, com um exemplar cada.

Comparando as espécies coletadas em bases de árvores, em relação as coletas em isca humana, encontramos que 11 espécies foram comuns nos dois tipos de coleta; 14 foram exclusivamente coletadas nas bases das árvores (Tabela 1) e outras 14 diferentes foram na isca humana, onde houve predominio das espécies do gênero Psychodopygus (Tab. 2), fato também confirmado por ARIAS \& FREITAS (1977) e FRAIHA et al. (1978), evidenciando que as espécies deste genêro são destacadamente antropófilas.

Das 52 espécies coletadas com armadilhas $\mathrm{CDC}, 32$ foram comuns em ambos os estratos; nas CDC colocadas a $1 \mathrm{~m}$ de altura foram coletadas exclusivamente 5 espécies, no entanto,
15 outras diferentes espécies foram capturadas exclusivamente nas armadilhas CDC colocadas a $6 \mathrm{~m}$ de altura.

A maior diversidade de espécies foi obtida com armadilhas luminosas colocadas a $6 \mathrm{~m}$ de altura; armadilhas semelhantes colocadas a $1 \mathrm{~m}$ de altura acima do solo deram como resultado uma frequência quantitativa sensívelmente menor de individuos.

Iguais resultados foram obtidos por ARIAS \& FREITAS (1982a, 1982b) em coletas realizadas na Reserva Florestal Ducke, e no Estado de Acre, e por BIANCARDI et al. (1982) em Rondônia; esses autores usaram a mesma metodología de trabalho.

Em virtude da diversidade de métodos empregados neste e em outros trabalhos de mesma natureza levados a efeito na região é díficil comparar. por exemplo, nossos resultados, aos de MARTINS \& SILVA (1964) ou de MARTINS, FALCÃO \& SILVA (1965). 


\section{AGRADECIMENTOS}

Este trabalho recebeu o apoio financeiro do UNDP/WORLD/BANK/ WHO, Special Programme for Research and Training in Tropical Diseases e do CNPq.

Agradecemos também ao $\mathrm{Sr}$. Artêmio Coêlho Da Silva pelo desenho do mapa e aos Srs. João F. Vidal e Raimundo N. Lima Santos pelo apoio no trabalho de campo.

\section{Bibliografia citada}

Arias, J.R. \& Freitas, R.A., 1977. Flebótomos da Amazônia Central do Brasil. I. Resultados obtidos das capturas com iscas humana e equina (Díp-tera:Psychodidae).Acta Amazonica, 7 (4): 507-527.

Arias, J.R. \& Freitas, R.A., 1982a. On the vectors of cutaneous leihsmaniasis in the Central Amazon, Brazil. 3. Phlebotomine sandfly stratification in a terra firme forest. Acta Amazonica, 12(3): 599-608.

Arias, J.R. \& Freitas, R.A., 1982b. The knowm geographical distribution of sandflies in the state of Acre, Brazil (Díptera : Psychodidae). Acta Amazonica, 12(2): 401-408.
Biancardi, C.B. Arias, J.R. Freitas, R.A. \& Castellón, E.G., 1982.The known geographical distributin of sandflies in the state of Rondônia, Brazil (Díptera: Psychodidae). Acta Amazonica. 12(1): 167-179.

Fraiha, H; Ward, R.D; Shàw, J.J. \& Lainson, R., 1978. Fauna antropófila de flebótomos da Rodovias Transamazonica, Brasil (Díptera : Psychodidae). Bol. Ofic. Sanit. Pan. Amer. 84(2): 134 - 139.

Martins, A.V. \& Silva, J.E., 1964. Notas sobre os flebótomos do Estado do Acre com a descrição de duas espécies novas (Díptera:Psychodidae), Revist, Brasil. Biol, 24(2):124 -138.

Martins, A.V.; Falcão, A.L. \& Silva, J.E., 1965. Notas sobre os flebótomos do territorio de Rondônia, com a descrição de seis espécies novas (Diptera : Psychodidae). Revst. Brasil. Biol, 25:1-20.

Martins, A.V.; Willians, P. \& Falcão, A.L., 1978. American Sandflies (Diptera:Psychodidae:Phlebotominae). Acad. Brasil. Ciências, Rio de Janeiro, 195p.

Ryan, L, 1986. Flebótomos do Estado do Pará, Brasil. Doc. Tec. $n^{\circ} 1$, Instituto Evandro Chagas, F.S.E.S.P., N.S., Belém, 153p.

Young, D.G., 1979, A review of the blood sucking Psychodid flies of Colômbia. AG. Exper. Stat. Univ. Florida, Ganeisville, U.S.A., 226p. 